TAIWANESE JOURNAL OF MATHEMATICS

Vol. 17, No. 5, pp. 1677-1691, October 2013

DOI: $10.11650 /$ tjm.17.2013.2440

This paper is available online at http://journal.taiwanmathsoc.org.tw

\title{
ON VECTOR GENERALIZATIONS OF VANDERMONDE'S CONVOLUTION
}

\author{
Yao Lin Ong
}

Abstract. In this paper, we introduce the vector generalizations of the well-known Vandermonde's convolution such as

$$
\begin{aligned}
& \sum_{\boldsymbol{j}=\boldsymbol{\alpha}_{\mathbf{1}}+\cdots+\boldsymbol{\alpha}_{\boldsymbol{k}}} \sum_{\boldsymbol{\ell}=\boldsymbol{\beta}_{1}+\cdots+\boldsymbol{\beta}_{\boldsymbol{k}}} \prod_{i=1}^{k}\left(\begin{array}{c}
\left|\boldsymbol{\alpha}_{\boldsymbol{i}}\right|+\left|\boldsymbol{\beta}_{\boldsymbol{i}}\right| \\
\left|\boldsymbol{\alpha}_{\boldsymbol{i}}\right|
\end{array}\right) M\left(\boldsymbol{\alpha}_{\boldsymbol{i}}\right) M\left(\boldsymbol{\beta}_{\boldsymbol{i}}\right) \\
= & M(\boldsymbol{j}) M(\boldsymbol{\ell})\left(\begin{array}{c}
|\boldsymbol{j}|+|\boldsymbol{\ell}|+k-1 \\
|\boldsymbol{j}|,|\boldsymbol{\ell}|, k-1
\end{array}\right),
\end{aligned}
$$

where $\boldsymbol{j}, \boldsymbol{\ell}, \boldsymbol{\alpha}_{\boldsymbol{i}}$ and $\boldsymbol{\beta}_{\boldsymbol{i}}$ are the vectors with nonnegative integer components, and $M(j)$ is the multinomial coefficient defined by $\left(\begin{array}{c}j_{1}+j_{2}+\cdots+j_{m} \\ j_{1}, \ldots, j_{m}\end{array}\right)$ with the value $\frac{|\boldsymbol{j}| !}{j_{1} ! \cdots j_{m} !}$. The main interest in such generalization comes from the number of multiple zeta values in the relations produced from the shuffle product of two sets of multiple zeta values in their iterated integral representations over simplices. Several generalizations of Vandermonde's convolution type are given as well.

\section{INTRODUCTION}

For a $r$-tuple of positive integers $\boldsymbol{\alpha}=\left(\alpha_{1}, \ldots, \alpha_{r}\right)$ with $\alpha_{r} \geq 2$, the multiple zeta value or $r$-fold Euler sum $\zeta(\boldsymbol{\alpha})$ is defined as [3]

$$
\zeta(\boldsymbol{\alpha})=\sum_{1 \leq n_{1}<n_{2}<\cdots<n_{r}} n_{1}^{-\alpha_{1}} n_{2}^{-\alpha_{2}} \cdots n_{r}^{-\alpha_{r}},
$$

The numbers $r$ and $|\boldsymbol{\alpha}|=\alpha_{1}+\cdots+\alpha_{r}$ are called the depth and weight of $\zeta(\boldsymbol{\alpha})$, respectively. Multiple zeta values can be represented by iterated integrals over simplices in the weighted-dimensional Euclidean space as $[2,3,13]$

$$
\int_{E_{|\boldsymbol{\alpha}|}} \Omega_{1} \cdots \Omega_{|\boldsymbol{\alpha}|} \text { or } \quad \int_{0}^{1} \Omega_{1} \cdots \Omega_{|\boldsymbol{\alpha}|}
$$

Received September 14, 2012, accepted March 31, 2013.

Communicated by Wen-Ching Li.

2010 Mathematics Subject Classification: Primary 40A25, 40B05; Secondary 11M41, 11M06, 33E20.

Key words and phrases: Vandermonde's convolution, Multiple zeta value, Shuffle product.

This research work is supported by the National Science Council of Taiwan. 
with the simplex $E_{|\boldsymbol{\alpha}|}$ defined by

$$
0<t_{1}<t_{2}<\cdots<t_{|\boldsymbol{\alpha}|}<1
$$

and

$$
\Omega_{j}= \begin{cases}\frac{d t_{j}}{1-t_{j}}, & \text { if } j=1, \alpha_{1}+1, \alpha_{1}+\alpha_{2}+1, \ldots, \alpha_{1}+\cdots+\alpha_{r-1}+1 \\ \frac{d t_{j}}{t_{j}}, & \text { otherwise. }\end{cases}
$$

In particular, we have for nonnegative integers $m$ and $n$ that

$$
\zeta\left(\{1\}^{m}, n+2\right)=\int_{E_{m+n+2}} \prod_{j=1}^{m+1} \frac{d t_{j}}{1-t_{j}} \prod_{k=m+2}^{m+n+2} \frac{d t_{k}}{t_{k}} .
$$

Note that the change of variables

$$
u_{1}=1-t_{m+n+2}, \quad u_{2}=1-t_{m+n+1}, \quad \ldots, \quad u_{m+n+2}=1-t_{1}
$$

yields the duality theorem $[10,13]$

$$
\zeta\left(\{1\}^{m}, n+2\right)=\zeta\left(\{1\}^{n}, m+2\right) .
$$

Once the multiple zeta values are expressed as iterated integrals, the shuffle product formula [2] of two multiple zeta values take the form

$$
\int_{0}^{1} \Omega_{1} \cdots \Omega_{m} \int_{0}^{1} \Omega_{m+1} \cdots \Omega_{m+n}=\sum_{\sigma} \int_{0}^{1} \Omega_{\sigma(1)} \cdots \Omega_{\sigma(m+n)},
$$

where $\sigma$ ranges over all $\left(\begin{array}{c}m+n \\ n\end{array}\right)$ permutations on the set $\{1,2, \ldots, m+n\}$ which preserves the orders of $\Omega_{1} \cdots \Omega_{m}$ and $\Omega_{m+1} \cdots \Omega_{m+n}$, i.e., for $1 \leq i<j \leq m$ or $m+1 \leq i<j \leq m+n$, we have $\sigma^{-1}(i)<\sigma^{-1}(j)$.

The following two propositions are essential to transform multiple zeta values into integrals, and vice versa.

Proposition 1.1. [5]. For nonnegative integers $m$ and $n$, we have

$$
\begin{aligned}
\zeta\left(\{1\}^{m}, n+2\right) & =\frac{1}{m !(n+1) !} \int_{0}^{1}\left(\log \frac{1}{1-t}\right)^{m}\left(\log \frac{1}{t}\right)^{n+1} \frac{d t}{1-t} \\
& =\frac{1}{m ! n !} \int_{0<t_{1}<t_{2}<1}\left(\log \frac{1}{1-t_{1}}\right)^{m}\left(\log \frac{1}{t_{2}}\right)^{n} \frac{d t_{1} d t_{2}}{\left(1-t_{1}\right) t_{2}} .
\end{aligned}
$$


Proposition 1.2. $[5,6]$. For nonnegative integers $p, q, r$, and $n$, we have

$$
\begin{aligned}
& \sum_{|\boldsymbol{\alpha}|=q+r+1} \zeta\left(\{1\}^{p}, \alpha_{0}, \ldots, \alpha_{q}+n+1\right) \\
= & \frac{1}{p ! q ! r ! n !} \int_{0<t_{1}<t_{2}<1}\left(\log \frac{1}{1-t_{1}}\right)^{p}\left(\log \frac{1-t_{1}}{1-t_{2}}\right)^{q}\left(\log \frac{t_{2}}{t_{1}}\right)^{r}\left(\log \frac{1}{t_{2}}\right)^{n} \frac{d t_{1} d t_{2}}{\left(1-t_{1}\right) t_{2}} .
\end{aligned}
$$

In particular, for integers $k, r \geq 0$, one has

$$
\sum_{|\boldsymbol{\alpha}|=k+r+1} \zeta\left(\alpha_{0}, \ldots, \alpha_{k-1}, \alpha_{k}+1\right)=\frac{1}{k ! r !} \int_{0<t_{1}<t_{2}<1}\left(\log \frac{1-t_{1}}{1-t_{2}}\right)^{k}\left(\log \frac{t_{2}}{t_{1}}\right)^{r} \frac{d t_{1} d t_{2}}{\left(1-t_{1}\right) t_{2}}
$$

Here we demonstrate an application of shuffle process of two multiple zeta values to reprove the well-known Euler decomposition formula as well as the Pascal's identity.

Theorem 1.3. For a pair of positive integers $p, q$, one has

$$
\zeta(p+1) \zeta(q+1)=\sum_{|\boldsymbol{\alpha}|=p+q+1}\left(\begin{array}{c}
\alpha_{2} \\
p
\end{array}\right) \zeta\left(\alpha_{1}, \alpha_{2}+1\right)+\sum_{|\boldsymbol{\alpha}|=p+q+1}\left(\begin{array}{c}
\alpha_{2} \\
q
\end{array}\right) \zeta\left(\alpha_{1}, \alpha_{2}+1\right) .
$$

Proof. By Proposition 1.1, we obtain the following integral expression

$$
\zeta(p+1) \zeta(q+1)=\frac{1}{p ! q !} \int_{0}^{1} \int_{0}^{1}\left(\log \frac{1}{t}\right)^{p}\left(\log \frac{1}{u}\right)^{q} \frac{d t}{1-t} \frac{d u}{1-u} .
$$

Subdivide the region of the integration into two simplices as

$$
D_{1}: 0<t<u<1 \text { and } D_{2}: 0<u<t<1 .
$$

On the simplex $D_{1}$, we replace the factor $\left(\log \frac{1}{t}\right)^{p}$ by its binomial expression

$$
\sum_{a=0}^{p} \frac{p !}{a !(p-a) !}\left(\log \frac{u}{t}\right)^{a}\left(\log \frac{1}{u}\right)^{p-a}
$$

so that the value of the integration over $D_{1}$ is

$$
\sum_{a=0}^{p}\left(\begin{array}{c}
p+q-a \\
q
\end{array}\right) \zeta(a+1, p+q-a+1)
$$

or equivalently,

$$
\sum_{|\boldsymbol{\alpha}|=p+q+1}\left(\begin{array}{c}
\alpha_{2} \\
q
\end{array}\right) \zeta\left(\alpha_{1}, \alpha_{2}+1\right)
$$


On the other hand, the value of the integration over $D_{2}$ is

$$
\sum_{b=0}^{q}\left(\begin{array}{c}
p+q-b \\
p
\end{array}\right) \zeta(b+1, p+q-b+1)
$$

or equivalently,

$$
\sum_{|\boldsymbol{\alpha}|=p+q+1}\left(\begin{array}{c}
\alpha_{2} \\
p
\end{array}\right) \zeta\left(\alpha_{1}, \alpha_{2}+1\right) .
$$

Our assertion then follows since the value of the integral (1.1) is equal to the sum of (1.2) and (1.3).

Remark 1.4. Theorem 1.3 is equivalent to the classical Euler's decomposition formula

$$
\zeta(s) \zeta(t)=\sum_{a=0}^{s-1}\left(\begin{array}{c}
a+t+1 \\
t-1
\end{array}\right) \zeta(t+a, s-a)+\sum_{a=0}^{t-1}\left(\begin{array}{c}
a+s+1 \\
s-1
\end{array}\right) \zeta(s+a, t-a)
$$

for any positive integers $s, t$ with $s \geq 2$ and $t \geq 2$ after some refinements in variables.

On the other hand, if we let $p=\ell+1$ and $q=r-\ell+1$ with $0 \leq \ell \leq r$, then we get

$$
\zeta(\ell+2) \zeta(r-\ell+2)=\sum_{|\boldsymbol{\alpha}|=r+3} \zeta\left(\alpha_{1}, \alpha_{2}+1\right)\left\{\left(\begin{array}{c}
\alpha_{2} \\
\ell+1
\end{array}\right)+\left(\begin{array}{c}
\alpha_{2} \\
r-\ell+1
\end{array}\right)\right\}
$$

The number of multiple zeta values appeared in the left-hand side of (1.4) must be equal to

$$
\left(\begin{array}{c}
(\ell+2)+(r-\ell+2) \\
\ell+2
\end{array}\right)=\left(\begin{array}{l}
r+4 \\
\ell+2
\end{array}\right) .
$$

In other words, we obtain the identity

$$
\sum_{\alpha=1}^{r+2}\left[\left(\begin{array}{c}
\alpha \\
\ell+1
\end{array}\right)+\left(\begin{array}{c}
\alpha \\
r-\ell+1
\end{array}\right)\right]=\left(\begin{array}{c}
r+4 \\
\ell+2
\end{array}\right)
$$

or with an elementary calculation that

$$
\left(\begin{array}{l}
r+3 \\
\ell+2
\end{array}\right)+\left(\begin{array}{l}
r+3 \\
\ell+1
\end{array}\right)=\left(\begin{array}{l}
r+4 \\
\ell+2
\end{array}\right),
$$

which is the well-known Pascal's identity. 


\section{Combinatorial Identities of Convolution Type}

The combinatorial identities of convolution type arose from the following identity developed in [4]:

$$
\sum_{a+b=k}\left(\begin{array}{c}
m+a \\
m
\end{array}\right)(\mu+1)^{b}=\frac{(m+k+1) !}{m ! k !} \int_{0}^{1} x^{m}(\mu+1-\mu x)^{k} d x,
$$

where $m$ and $k$ are nonnegative integers, and $\mu$ is a complex number. By comparing the coefficient of $\mu^{j}(0 \leq j \leq k)$ on both sides of the above identity, we obtain the following formula involving the binomial coefficient:

$$
\sum_{a+b=k}\left(\begin{array}{c}
m+a \\
m
\end{array}\right)\left(\begin{array}{l}
b \\
j
\end{array}\right)=\left(\begin{array}{c}
m+k+1 \\
m+j+1
\end{array}\right)
$$

which is quite similar to the classical identity appeared on page 169 of [8], given as

$$
\sum_{0 \leq k \leq \ell}\left(\begin{array}{c}
\ell-k \\
m
\end{array}\right)\left(\begin{array}{c}
q+k \\
n
\end{array}\right)=\left(\begin{array}{c}
\ell+q+1 \\
m+n+1
\end{array}\right)
$$

with integers $\ell, m \geq 0$ and $n \geq q \geq 0$. Both identities can be restated as

$$
\sum_{a+b=k}\left(\begin{array}{c}
m+a \\
m
\end{array}\right)\left(\begin{array}{c}
n+b \\
n
\end{array}\right)=\left(\begin{array}{c}
m+n+k+1 \\
m+n+1
\end{array}\right)
$$

with integers $k, m, n \geq 0$. As a matter of fact, such identity came from the following widely known Vandermonde's convolution

$$
\sum_{k=0}^{r}\left(\begin{array}{c}
m \\
k
\end{array}\right)\left(\begin{array}{c}
n \\
r-k
\end{array}\right)=\left(\begin{array}{c}
m+n \\
r
\end{array}\right), \quad m, n, r \in \mathbb{N} \cup\{0\},
$$

which was named after Alexandre-Théophile Vandermonde in the late 1700s [1]. However, it was also appeared in a book written by Chu Shih-Chieh in China around 1303.

Differentiate both sides of the well-known geometry series expansion

$$
\frac{1}{1-x}=\sum_{a=0}^{\infty} x^{a}, \quad|x|<1
$$

$m$ times, then we get

$$
\frac{1}{(1-x)^{m+1}}=\sum_{a=0}^{\infty}\left(\begin{array}{c}
m+a \\
m
\end{array}\right) x^{a}, \quad|x|<1 .
$$

So the sum of products of binomial coefficients on the left hand side of (2.3) is just the coefficient of $x^{k}$ in the product

$$
\frac{1}{(1-x)^{m+1}} \cdot \frac{1}{(1-x)^{n+1}}
$$

when both of them are expanded in power series. Therefore, the assertion (2.3) is proved and it has further extensions in the following. 
Proposition 2.1. For nonnegative integers $j, \beta_{1}, \beta_{2}, \ldots, \beta_{k}$ with $\ell=\beta_{1}+\beta_{2}+$ $\ldots+\beta_{k}$, we have

$$
\sum_{|\boldsymbol{\alpha}|=j}\left(\begin{array}{c}
\alpha_{1}+\beta_{1} \\
\beta_{1}
\end{array}\right)\left(\begin{array}{c}
\alpha_{2}+\beta_{2} \\
\beta_{2}
\end{array}\right) \cdots\left(\begin{array}{c}
\alpha_{k}+\beta_{k} \\
\beta_{k}
\end{array}\right)=\left(\begin{array}{c}
j+\ell+k-1 \\
\ell+k-1
\end{array}\right) .
$$

Proof. The sum of products of $k$ binomial coefficients is just the coefficient of $x^{j}$ in the product

$$
\prod_{i=1}^{k} \frac{1}{(1-x)^{\beta_{i}+1}}
$$

since

$$
\frac{1}{(1-x)^{\beta_{i}+1}}=\sum_{\alpha_{i}=0}^{\infty}\left(\begin{array}{c}
\alpha_{i}+\beta_{i} \\
\beta_{i}
\end{array}\right) x^{\alpha_{i}}, \quad i=1,2, \ldots, k .
$$

By the power series expansion of the function

$$
\frac{1}{(1-x)^{\ell+k}}
$$

we get our assertion.

Remark 2.2. [11]. If we also sum over all $\beta_{1}, \beta_{2}, \ldots, \beta_{n}$ with $\beta_{1}+\beta_{2}+\ldots+\beta_{n}=\ell$, then we get

$$
\sum_{|\boldsymbol{\alpha}|=j} \sum_{|\boldsymbol{\beta}|=\ell}\left(\begin{array}{c}
\alpha_{1}+\beta_{1} \\
\alpha_{1}
\end{array}\right)\left(\begin{array}{c}
\alpha_{2}+\beta_{2} \\
\alpha_{2}
\end{array}\right) \cdots\left(\begin{array}{c}
\alpha_{k}+\beta_{k} \\
\alpha_{k}
\end{array}\right)=\left(\begin{array}{c}
j+\ell+k-1 \\
j, \ell, k-1
\end{array}\right),
$$

where $\left(\begin{array}{c}j+\ell+k-1 \\ j, \ell, k-1\end{array}\right)$ is the multinomial coefficient defined by $\frac{(j+\ell+k-1) !}{j ! \ell !(k-1) !}$.

Vandermonde's convolution (2.2) and its general forms are useful in counting the numbers of multiple zeta values produced from the shuffle process of multiple zeta values through their integral representations. Moreover, they can be extended in vector forms, which are difficult to be proved straightforwardly.

For simplicity, we adopt the following vector notations throughout this paper. For a vector $\boldsymbol{g}=\left(g_{1}, g_{2}, \ldots, g_{n}\right)$ with nonnegative integer components, denote

$$
\begin{gathered}
|\boldsymbol{g}|=g_{1}+\cdots+g_{n}, \\
\boldsymbol{g} !=g_{1} ! \cdots g_{n} !
\end{gathered}
$$

and

$$
M(\boldsymbol{g})=\left(\begin{array}{c}
g_{1}+g_{2}+\ldots+g_{n} \\
g_{1}, g_{2}, \ldots, g_{n}
\end{array}\right), \text { the multinomial coefficient }
$$


respectively.

Now, let us introduce new combinatorial identities of Vandermonde's convolution type. First of all, consider the integral

$$
\frac{1}{m ! k ! n !} \iint_{0<t_{1}<t_{2}<1}\left(\log \frac{1}{1-t_{1}}\right)^{m}\left(\log \frac{1}{1-t_{2}}\right)^{k}\left(\log \frac{t_{2}}{t_{1}}\right)^{n} \frac{d t_{1}}{\left(1-t_{1}\right)} \frac{d t_{2}}{t_{2}}
$$

for nonnegative integers $m, k$, and $n$. As

$$
\begin{aligned}
\left(\log \frac{1}{1-t_{2}}\right)^{k} & =\left(\log \frac{1}{1-t_{1}}+\log \frac{1-t_{1}}{1-t_{2}}\right)^{k} \\
& =\sum_{a+b=k} \frac{k !}{a ! b !}\left(\log \frac{1}{1-t_{1}}\right)^{a}\left(\log \frac{1-t_{1}}{1-t_{2}}\right)^{b},
\end{aligned}
$$

the sum of multiple zeta values represented by the integral (2.8) is

$$
\sum_{a+b=k}\left(\begin{array}{c}
m+a \\
m
\end{array}\right) \sum_{|\boldsymbol{\alpha}|=b+n+1} \zeta\left(\{1\}^{m+a}, \alpha_{0}, \ldots, \alpha_{b}+1\right)
$$

in light of Proposition 1.2. Therefore, the number of multiple zeta values in (2.9) is

$$
\sum_{a+b=k}\left(\begin{array}{c}
m+a \\
m
\end{array}\right)\left(\begin{array}{c}
n+b \\
n
\end{array}\right)
$$

or equivalently,

$$
\left(\begin{array}{c}
m+k+n+1 \\
k
\end{array}\right)
$$

by the help of (2.2).

However, if we replace the factor

$$
\frac{1}{k !}\left(\log \frac{1}{1-t_{2}}\right)^{k}
$$

of the integral (2.8) by its vector form

$$
\frac{1}{\boldsymbol{k} !}\left(\log \frac{1}{1-t_{2}}\right)^{|\boldsymbol{k}|}
$$

where $\boldsymbol{k}=\left(k_{1}, k_{2}, \ldots, k_{r}\right)$ is a vector with nonnegative integer components, then there would be at least two ways to count the number of multiple zeta values.

If we treat the factor (2.11) as only one factor, then the number of multiple zeta values of the integral (2.8) is

$$
M(\boldsymbol{k}) \sum_{a+b=|\boldsymbol{k}|}\left(\begin{array}{c}
m+a \\
m
\end{array}\right)\left(\begin{array}{c}
n+b \\
n
\end{array}\right) .
$$


On the other hand, if we replace each component

$$
\left(\log \frac{1}{1-t_{2}}\right)^{k_{j}}
$$

of the factor (2.11) by its binomial expansion as

$$
\sum_{\alpha_{j}+\beta_{j}=k_{j}} \frac{k_{j} !}{\alpha_{j} ! \beta_{j} !}\left(\log \frac{1}{1-t_{1}}\right)^{\alpha_{j}}\left(\log \frac{1-t_{1}}{1-t_{2}}\right)^{\beta_{j}},
$$

$j=1,2, \ldots, r$, then the number of multiple zeta values of the integral (2.8) is

$$
\sum_{\boldsymbol{\alpha}+\boldsymbol{\beta}=\boldsymbol{k}}\left(\begin{array}{c}
m+\alpha_{1}+\alpha_{2}+\ldots+\alpha_{r} \\
m, \alpha_{1}, \alpha_{2}, \ldots, \alpha_{r}
\end{array}\right)\left(\begin{array}{c}
n+\beta_{1}+\beta_{2}+\ldots+\beta_{r} \\
n, \beta_{1}, \beta_{2}, \ldots, \beta_{r}
\end{array}\right) .
$$

This leads to the following nontrivial combinatorial identity of convolution type.

Theorem 2.3. Let $\boldsymbol{k}=\left(k_{1}, k_{2}, \ldots, k_{r}\right)$ be a vector with nonnegative integer components. Then for nonnegative integers $m$ and $n$, we have

$$
\begin{array}{r}
\sum_{\boldsymbol{\alpha}+\boldsymbol{\beta}=\boldsymbol{k}}\left(\begin{array}{c}
m+|\boldsymbol{\alpha}| \\
m, \boldsymbol{\alpha}
\end{array}\right)\left(\begin{array}{c}
n+|\boldsymbol{\beta}| \\
n, \boldsymbol{\beta}
\end{array}\right) \\
=M(\boldsymbol{k})\left(\begin{array}{c}
m+|\boldsymbol{k}|+n+1 \\
|\boldsymbol{k}|
\end{array}\right),
\end{array}
$$

where the vectors $\boldsymbol{\alpha}$ and $\boldsymbol{\beta}$ are r-tuples of nonnegative integers as the vector $\boldsymbol{k}$.

\section{Applications of Vandermonde's Convolution to Shuffle Relations}

The shuffle product of two multiple zeta values of weight $m$ and $n$, respectively, will produce $\left(\begin{array}{c}m+n \\ n\end{array}\right)$ multiple zeta values of weight $m+n$. If we are able to count the total numbers of multiple zeta values produced from a shuffle product of two multiple zeta values, then we obtain an identity among binomial coefficients, which might be of interest to combinatorics. This motivates us to focus on the integrals arising from the shuffle product of two sets of multiple zeta values of the form $\zeta\left(\{1\}^{m}, n+2\right)$, or the so-called multiple zeta values of height one, since their double integral representations over simplicies of dimension two in Proposition 1.1 are critical to proceed with the shuffle process. We shall introduce another vector generalization of Vandermonde's convolution in the following.

For integers $k, r, j, \ell$ with $0 \leq j \leq k$ and $0 \leq \ell \leq r$, let us consider the integral

$$
\begin{aligned}
& \frac{1}{j !(k-j) !(\ell+1) !(r-\ell+1) !} \\
& \times \iint_{0<t_{1}<t_{2}<1}\left(\log \frac{1}{1-t_{1}}\right)^{k-j}\left(\log \frac{1}{t_{1}}\right)^{\ell+1}\left(\log \frac{1}{1-t_{2}}\right)^{j}\left(\log \frac{1}{t_{2}}\right)^{r-\ell+1} \frac{d t_{1}}{1-t_{1}} \frac{d t_{2}}{1-t_{2}}
\end{aligned}
$$


which represents the product of two multiple zeta values

$$
\zeta\left(\{1\}^{k-j}, \ell+2\right) \zeta\left(\{1\}^{j}, r-\ell+2\right)
$$

in light of Proposition 1.1. Replace the factor

$$
\left(\log \frac{1}{t_{1}}\right)^{\ell+1} \text { and }\left(\log \frac{1}{1-t_{2}}\right)^{j}
$$

by their binomial expansions

$$
\begin{gathered}
\sum_{c+d=\ell+1} \frac{(\ell+1) !}{c ! d !}\left(\log \frac{t_{2}}{t_{1}}\right)^{c}\left(\log \frac{1}{t_{2}}\right)^{d} \text { and } \\
\sum_{a+b=j} \frac{j !}{a ! b !}\left(\log \frac{1}{1-t_{1}}\right)^{a}\left(\log \frac{1-t_{1}}{1-t_{2}}\right)^{b}
\end{gathered}
$$

respectively, we see that the number of multiple zeta values represented by the integral $(3.1)$ is

$$
\sum_{a+b=j}\left(\begin{array}{c}
k-j+a \\
k-j
\end{array}\right) \sum_{c+d=\ell+1}\left(\begin{array}{c}
d+r-\ell+1 \\
r-\ell+1
\end{array}\right)\left(\begin{array}{c}
c+b \\
b
\end{array}\right) .
$$

Apply Vandermonde's convolution (2.2) to the inner sum, the number (3.2) then turns out to be

$$
\sum_{a+b=j}\left(\begin{array}{c}
k-j+a \\
k-j
\end{array}\right)\left(\begin{array}{c}
b+r+3 \\
\ell+1
\end{array}\right)
$$

or simply

$$
\sum_{a+b=k}\left(\begin{array}{c}
a \\
k-j
\end{array}\right)\left(\begin{array}{c}
b+r+3 \\
\ell+1
\end{array}\right)
$$

On the other hand, let

$$
\boldsymbol{j}=\left(j_{1}, j_{2}, \ldots, j_{m}\right) \text { and } \boldsymbol{\ell}=\left(\ell_{1}, \ell_{2}, \ldots, \ell_{n}\right)
$$

be two vectors of nonnegative integer components together with the symbols

$$
j !=j_{1} ! \cdots j_{m} ! \quad \text { and } \quad \ell !=\ell_{1} ! \cdots \ell_{n} !
$$

as mentioned earlier.

Consider the integral

$$
\begin{aligned}
& \frac{1}{\boldsymbol{j} !(k-j) ! \ell !(r-\ell+1) !} \times \\
& \iint_{0<t_{1}<t_{2}<1}\left(\log \frac{1}{1-t_{1}}\right)^{k-j}\left(\log \frac{1}{t_{1}}\right)^{|\boldsymbol{\ell}|+1}\left(\log \frac{1}{1-t_{2}}\right)^{|\boldsymbol{j}|}\left(\log \frac{1}{t_{2}}\right)^{r-\ell+1} \frac{d t_{1}}{1-t_{1}} \frac{d t_{2}}{1-t_{2}} .
\end{aligned}
$$


Then the number of multiple zeta values represented by (3.3) is given by

$$
\sum_{\boldsymbol{\alpha}+\boldsymbol{\beta}=\boldsymbol{j}} \sum_{\boldsymbol{\gamma}+\boldsymbol{\delta}=\boldsymbol{\ell}}\left(\begin{array}{c}
|\boldsymbol{\alpha}|+k-j \\
\boldsymbol{\alpha}, k-j
\end{array}\right)\left(\begin{array}{c}
|\boldsymbol{\beta}|+|\boldsymbol{\gamma}| \\
\boldsymbol{\beta}, \boldsymbol{\gamma}
\end{array}\right)\left(\begin{array}{c}
|\boldsymbol{\delta}|+r-\ell+1 \\
\boldsymbol{\delta}, r-\ell+1
\end{array}\right)
$$

or

$$
\sum_{\boldsymbol{\alpha}+\boldsymbol{\beta}=\boldsymbol{j}} \sum_{\boldsymbol{\gamma}+\boldsymbol{\delta}=\ell}\left(\begin{array}{c}
|\boldsymbol{\alpha}|+k-j \\
|\boldsymbol{\alpha}|
\end{array}\right)\left(\begin{array}{c}
|\boldsymbol{\beta}|+|\boldsymbol{\gamma}| \\
|\boldsymbol{\beta}|
\end{array}\right)\left(\begin{array}{c}
|\boldsymbol{\delta}|+r-\ell+1 \\
|\boldsymbol{\delta}|
\end{array}\right) M(\boldsymbol{\alpha}) M(\boldsymbol{\beta}) M(\boldsymbol{\gamma}) M(\boldsymbol{\delta}) .
$$

Again, two different expressions for the number of multiple zeta values represented by (3.3) leads to the following combinatorial identity.

Theorem 3.1. For integers $k, r, j, \ell$ with $0 \leq j \leq k$ and $0 \leq \ell \leq r$, we have

$$
\begin{aligned}
& \sum_{\boldsymbol{\alpha}+\boldsymbol{\beta}=\boldsymbol{j} \boldsymbol{\gamma}+\boldsymbol{\delta}=\ell}\left(\begin{array}{c}
|\boldsymbol{\alpha}|+k-j \\
|\boldsymbol{\alpha}|
\end{array}\right)\left(\begin{array}{c}
|\boldsymbol{\beta}|+|\boldsymbol{\gamma}| \\
|\boldsymbol{\beta}|
\end{array}\right)\left(\begin{array}{c}
|\boldsymbol{\delta}|+r-\ell+1 \\
|\boldsymbol{\delta}|
\end{array}\right) M(\boldsymbol{\alpha}) M(\boldsymbol{\beta}) M(\boldsymbol{\gamma}) M(\boldsymbol{\delta}) \\
& =M(\boldsymbol{j}) M(\boldsymbol{\ell}) \sum_{a+b=k}\left(\begin{array}{c}
a \\
k-j
\end{array}\right)\left(\begin{array}{c}
b+r+3 \\
\ell+1
\end{array}\right),
\end{aligned}
$$

where the vectors $\boldsymbol{\alpha}$ and $\boldsymbol{\beta}$ are $m$-tuples of nonnegative integers as the vector $\boldsymbol{j}$, and the vectors $\gamma$ and $\boldsymbol{\delta}$ are $n$-tuples of nonnegative integers as the vector $\ell$.

For further generalizations of Vandermonde's convolution of vector type, we are going to carry out the shuffle product of

$$
\zeta\left(\{1\}^{p}, q+\ell+2\right) \quad \text { and } \quad \zeta\left(\{1\}^{m}, n+2\right)
$$

through their integral representations

$$
\frac{1}{p ! q ! \ell !} \int_{0<t_{1}<t_{2}<1}\left(\log \frac{1}{1-t_{2}}\right)^{p}\left(\log \frac{t_{2}}{t_{1}}\right)^{q}\left(\log \frac{1}{t_{2}}\right)^{\ell} \frac{d t_{1}}{\left(1-t_{1}\right)} \frac{d t_{2}}{t_{2}}
$$

and

$$
\frac{1}{m !(n+1) !} \int_{0}^{1}\left(\log \frac{1}{1-u}\right)^{m}\left(\log \frac{1}{u}\right)^{n+1} \frac{d u}{1-u}
$$

with nonnegative integers $p, q, \ell, m$, and $n$. Note that the weights of these two multiple zeta values are $w_{1}=p+q+\ell+2$ and $w_{2}=m+n+2$. Therefore, it will produce

$$
\left(\begin{array}{c}
w_{1}+w_{2} \\
w_{1}
\end{array}\right)
$$

multiple zeta values of weight $w_{1}+w_{2}$. 
As the replacement of the shuffle process, we decompose the domain $D$ of the shuffle product of (3.5) and (3.6) into 3 simplicies, namely,

$$
\begin{array}{ll}
D_{1}: & 0<t_{1}<t_{2}<u<1, \\
D_{2}: & 0<t_{1}<u<t_{2}<1 \\
D_{3}: & 0<u<t_{1}<t_{2}<1 .
\end{array} \text { and }
$$

On the shuffle process over the simplex $D_{1}: 0<t_{1}<t_{2}<u<1$, we replace the factors

$$
\left(\log \frac{1}{t_{2}}\right)^{\ell} \text { and }\left(\log \frac{1}{1-u}\right)^{m}
$$

by

$$
\left(\log \frac{u}{t_{2}}+\log \frac{1}{u}\right)^{\ell}
$$

and

$$
\left(\log \frac{1}{1-t_{1}}+\log \frac{1-t_{1}}{1-t_{2}}+\log \frac{1-t_{2}}{1-u}\right)^{m}
$$

respectively.

Expand them into multinomial expansions, then the number of multiple zeta values obtained from the evaluations of the integrals over $D_{1}$ is given by

$$
\sum_{m_{1}+m_{2}+m_{3}=m} \sum_{\ell_{1}+\ell_{2}=\ell}\left(\begin{array}{c}
m_{1}+p \\
p
\end{array}\right)\left(\begin{array}{c}
m_{2}+q \\
q
\end{array}\right)\left(\begin{array}{c}
m_{3}+\ell_{1} \\
\ell_{1}
\end{array}\right)\left(\begin{array}{c}
\ell_{2}+n+1 \\
n+1
\end{array}\right) .
$$

By summing over $m_{1}+m_{2}+m_{3}=m$, the number (3.8) becomes

$$
\sum_{\ell_{1}+\ell_{2}=\ell}\left(\begin{array}{c}
\ell_{1}+m+p+q+2 \\
m
\end{array}\right)\left(\begin{array}{c}
\ell_{2}+n+1 \\
n+1
\end{array}\right) \text {. }
$$

In a similar manner, the numbers of multiple zeta values obtained from the evaluations of the integrals over the simplicies $D_{2}: 0<t_{1}<u<t_{2}<1$ and $D_{3}: 0<u<t_{1}<t_{2}<1$ are given by

$$
\sum_{a+b=q}\left(\begin{array}{c}
a+m+p+1 \\
m
\end{array}\right)\left(\begin{array}{c}
b+n+\ell+2 \\
n+1
\end{array}\right)
$$

and

$$
\sum_{p_{1}+p_{2}=p}\left(\begin{array}{c}
p_{1}+m \\
m
\end{array}\right)\left(\begin{array}{c}
p_{2}+q+\ell+n+3 \\
n+1
\end{array}\right)
$$

respectively. 
In a word, if we express (3.7) as

$$
\sum_{\alpha_{1}+\alpha_{2}=w_{1}}\left(\begin{array}{c}
\alpha_{1}+m \\
m
\end{array}\right)\left(\begin{array}{c}
\alpha_{2}+n+1 \\
n+1
\end{array}\right)
$$

in light of (2.3), then it is divided into three parts corresponding to

$$
0 \leq \alpha_{1} \leq p, \quad p+1 \leq \alpha_{1} \leq p+q+1, \quad p+q+2 \leq \alpha_{1} \leq p+q+\ell+2 .
$$

That is, we conclude that

$$
\begin{aligned}
& \sum_{\alpha_{1}+\alpha_{2}=p+q+\ell+2}\left(\begin{array}{c}
\alpha_{1}+m \\
m
\end{array}\right)\left(\begin{array}{c}
\alpha_{2}+n+1 \\
n+1
\end{array}\right) \\
= & \sum_{p_{1}+p_{2}=p}\left(\begin{array}{c}
p_{1}+m \\
m
\end{array}\right)\left(\begin{array}{c}
p_{2}+q+\ell+n+3 \\
n+1
\end{array}\right)+\sum_{a+b=q}\left(\begin{array}{c}
a+m+p+1 \\
m
\end{array}\right)\left(\begin{array}{c}
b+n+\ell+2 \\
n+1
\end{array}\right) \\
& +\sum_{\ell_{1}+\ell_{2}=\ell}\left(\begin{array}{c}
\ell_{1}+m+p+q+2 \\
m
\end{array}\right)\left(\begin{array}{c}
\ell_{2}+n+1 \\
n+1
\end{array}\right)
\end{aligned}
$$

for nonnegative integers $p, q, \ell, m, n$.

Replace the factor $\frac{1}{q !}\left(\log \frac{t_{2}}{t_{1}}\right)^{q}$ by its vector form $\frac{1}{q !}\left(\log \frac{t_{2}}{t_{1}}\right)^{|\boldsymbol{q}|}$ in the shuffle product of (3.5) and (3.6) over the simplex $D_{2}$, and count the number of multiple zeta values in the resulted integral, the following vector generalization of convolution type is obtained at hand.

Theorem 3.2. Suppose that $p, \ell, m, n$ are nonnegative integers and $\boldsymbol{q}=\left(q_{1}, q_{2}, \ldots\right.$, $\left.q_{r}\right)$ is a vector with nonnegative integer components. Then

$$
\begin{gathered}
\sum_{\boldsymbol{\alpha}+\boldsymbol{\beta}=\boldsymbol{q}}\left(\begin{array}{c}
|\boldsymbol{\alpha}|+m+p+1 \\
m
\end{array}\right) M(\boldsymbol{\alpha}) M(\boldsymbol{\beta})\left(\begin{array}{c}
|\boldsymbol{\beta}|+n+\ell+2 \\
n+1
\end{array}\right) \\
\quad=M(\boldsymbol{q}) \sum_{a+b=q}\left(\begin{array}{c}
a+m+p+1 \\
m
\end{array}\right)\left(\begin{array}{c}
b+n+\ell+2 \\
n+1
\end{array}\right),
\end{gathered}
$$

where the vectors $\boldsymbol{\alpha}$ and $\boldsymbol{\beta}$ are r-tuples of nonnegative integers as the vector $\boldsymbol{q}$.

\section{Further Generalizations}

For the final remark, we are able to obtain various vector generalizations of convolution type through different vector decompositions provided that we exchange the suitable integer exponents of particular terms into vectors in the iterated integral representations of multiple zeta values. For example, the number of multiple zeta values in the following integral

(4.1) $\frac{1}{j ! \ell !} \iint_{\substack{0<t_{1}<t_{2}<1 \\ 0<u_{1}<u_{2}<1}}\left(\log \frac{1-t_{1}}{1-t_{2}}-\log \frac{1-u_{1}}{1-u_{2}}\right)^{j}\left(\log \frac{t_{2}}{t_{1}}-\log \frac{u_{2}}{u_{1}}\right)^{\ell} \frac{d t_{1}}{1-t_{1}} \frac{d t_{2}}{t_{2}} \frac{d u_{1}}{1-u_{1}} \frac{d u_{2}}{u_{2}}$ 
for nonnegative integers $j$ and $\ell$ is given by

$$
\sum_{a+b=j} \sum_{c+d=\ell}(-1)^{b+d}\left(\begin{array}{c}
a+c \\
a
\end{array}\right)\left(\begin{array}{c}
b+d \\
b
\end{array}\right)
$$

since such an integral is separable and it could be expressed in terms of sums of products of multiple zeta values as

$\sum_{a+b=j} \sum_{c+d=\ell}(-1)^{b+d} \sum_{|\alpha|=a+c+1} \zeta\left(\alpha_{0}, \ldots, \alpha_{a-1}, \alpha_{a}+1\right) \sum_{|\beta|=b+d+1} \zeta\left(\beta_{0}, \ldots, \beta_{b-1}, \alpha_{b}+1\right)$

by the help of Proposition 1.2. However, the number (4.2) also comes from the coefficient of $x^{\ell}$ in the power series expansion at $x=0$ of the function

$$
\sum_{a+b=j}(-1)^{b} \frac{1}{(1-x)^{a+1}} \cdot \frac{1}{(1+x)^{b+1}}
$$

This leads to the following combinatorial identity involving binomial coefficients

$$
\sum_{a+b=j} \sum_{c+d=\ell}(-1)^{b+d}\left(\begin{array}{c}
a+c \\
a
\end{array}\right)\left(\begin{array}{c}
b+d \\
b
\end{array}\right)=\frac{1}{2}\left(1+(-1)^{j+\ell}\right)\left(\begin{array}{c}
j+\ell \\
j
\end{array}\right)
$$

in light of the equivalent expression of (4.3)

$$
\frac{1}{2}\left(\frac{1}{(1-x)^{j+1}}+\frac{(-1)^{j}}{(1+x)^{j+1}}\right) .
$$

Again, replacing the exponents $j$ and $\ell$ in the integral (4.1) by their vector forms respectively gives another vector generalization of the combinatorial identity (4.4).

Theorem 4.1. Let $\boldsymbol{j}=\left(j_{1}, \ldots, j_{m}\right)$ and $\boldsymbol{\ell}=\left(\ell_{1}, \ldots, \ell_{n}\right)$ be two vectors of nonnegative integer components. Then we have

$$
\begin{aligned}
& \sum_{\boldsymbol{\alpha}+\boldsymbol{\beta}=\boldsymbol{j}} \sum_{\boldsymbol{\gamma}+\boldsymbol{\delta}=\ell}(-1)^{|\boldsymbol{\beta}|+|\boldsymbol{\delta}|}\left(\begin{array}{c}
|\boldsymbol{\alpha}|+|\boldsymbol{\gamma}| \\
\boldsymbol{\alpha}, \boldsymbol{\gamma}
\end{array}\right)\left(\begin{array}{c}
|\boldsymbol{\beta}|+|\boldsymbol{\delta}| \\
\boldsymbol{\beta}, \boldsymbol{\delta}
\end{array}\right) \\
= & \frac{1}{2} M(\boldsymbol{j}) M(\boldsymbol{\ell})\left(1+(-1)^{|\boldsymbol{j}|+|\boldsymbol{\ell}|}\right)\left(\begin{array}{c}
|\boldsymbol{j}|+|\boldsymbol{\ell}| \\
|\boldsymbol{j}|
\end{array}\right),
\end{aligned}
$$

where the vectors $\boldsymbol{\alpha}$ and $\boldsymbol{\beta}$ are m-tuples of nonnegative integers as the vector $\boldsymbol{j}$, and the vectors $\gamma$ and $\boldsymbol{\delta}$ are $n$-tuples of nonnegative integers as the vector $\boldsymbol{\ell}$.

Finally, the generalizations of Vandermonde's convolution (2.6) and (2.7) are both generalized to vector types in the following. 
Theorem 4.2. Let $\boldsymbol{j}=\left(j_{1}, j_{2}, \ldots, j_{m}\right)$ be a vector with nonnegative integer components. Then for any positive integer $k \geq 2$, we have

$$
\sum_{\boldsymbol{j}=\boldsymbol{\alpha}_{\mathbf{1}}+\cdots+\boldsymbol{\alpha}_{k}} M\left(\boldsymbol{\alpha}_{\mathbf{1}}\right) M\left(\boldsymbol{\alpha}_{\mathbf{2}}\right) \cdots M\left(\boldsymbol{\alpha}_{\boldsymbol{k}}\right)=M(\boldsymbol{j})\left(\begin{array}{c}
|\boldsymbol{j}|+k-1 \\
|\boldsymbol{j}|
\end{array}\right),
$$

where the vectors $\boldsymbol{\alpha}_{\boldsymbol{i}}$ 's are m-tuples of nonnegative integers as the vector $\boldsymbol{j}$.

Theorem 4.3. Let $\boldsymbol{j}=\left(j_{1}, j_{2}, \ldots, j_{m}\right)$ and $\boldsymbol{\ell}=\left(\ell_{1}, \ell_{2}, \ldots, \ell_{n}\right)$ be a pair of vectors with nonnegative integer components. Then for any positive integer $k \geq 2$, we have

$$
\begin{aligned}
& \sum_{\boldsymbol{j}=\boldsymbol{\alpha}_{\mathbf{1}}+\cdots+\boldsymbol{\alpha}_{\boldsymbol{k}}} \sum_{\boldsymbol{\ell}=\boldsymbol{\beta}_{\mathbf{1}}+\cdots+\boldsymbol{\beta}_{\boldsymbol{k}}} \prod_{i=1}^{k}\left(\begin{array}{c}
\left|\boldsymbol{\alpha}_{\boldsymbol{i}}\right|+\left|\boldsymbol{\beta}_{\boldsymbol{i}}\right| \\
\left|\boldsymbol{\alpha}_{\boldsymbol{i}}\right|
\end{array}\right) M\left(\boldsymbol{\alpha}_{\boldsymbol{i}}\right) M\left(\boldsymbol{\beta}_{\boldsymbol{i}}\right) \\
= & M(\boldsymbol{j}) M(\boldsymbol{\ell})\left(\begin{array}{c}
|\boldsymbol{j}|+|\boldsymbol{\ell}|+k-1 \\
|\boldsymbol{j}|,|\boldsymbol{\ell}|, k-1
\end{array}\right),
\end{aligned}
$$

where the vectors $\boldsymbol{\alpha}_{\boldsymbol{i}}$ 's are $m$-tuples of nonnegative integers as the vector $\boldsymbol{j}$, and the vectors $\boldsymbol{\beta}_{\boldsymbol{i}}$ 's are $n$-tuples of nonnegative integers as the vector $\boldsymbol{\ell}$.

\section{ACKNOWLEDGMENTS}

The author is grateful to Professor Minking Eie for the helpful ideas pertaining to this work in the study of the theory of multiple zeta values with applications in combinatorics. The sincere gratitude is also due to the referee for the valuable suggestions and comments which led to several improvements of this paper.

\section{REFERENCES}

1. Richard Askey, Orthogonal polynomials and special functions, Regional Conference Series in Applied Mathematics, 21 (1975), Philadelphia, PA: SIAM, viii+110.

2. J. M. Borwein, D. M. Bradley, D. J. Broadhurst and P. Lisoněk, Special values of multiple polylogarithms, Trans. Amer. Math. Soc., 353(3) (2001), 907-941.

3. D. Bowman and D. M. Bradley, Multiple polylogarithms: a brief survey, Contemp. Math., 291 (2001), 71-92.

4. M. Eie, T.-Y. Lee and Y. L. Ong, Applications of shuffle products of multiple zeta values in combinatorics, J. Comb. Number Theory, 4(3) (2012), 65-81.

5. M. Eie, W.-C. Liaw and Y. L. Ong, A restricted sum formula among multiple zeta values, J. Number Theory, 129(4) (2009), 908-921.

6. M. Eie and C.-S. Wei, A short proof for the sum formula and its generalization, Arch. Math., 91(4) (2008), 330-338. 
7. L. Euler, Meditationes circa singulare serierum genus, Novi Comm. Acad. Sci. Petropol., 20 (1775), 140-186; (Reprinted in Opera Omnia, Ser. I, no. 15, 217-267, Teubner, Berlin, 1927).

8. R. L. Graham, D. E. Knuth and O. Patashnik, Concrete Mathematics: A Foundation for Computer Science, Addison-Wesley Publishing Company, Reading, 1989.

9. A. Granville, A decomposition of Riemann's zeta-function, Analytic Number Theory, Kyoto, 1996, 95-101, London Math. Soc. Lecture Note Ser. 247, Cambridge Univ. Press, Cambridge, 1997.

10. M. E. Hoffman, Multiple harmonic series, Pacific J. Math., 152(2) (1992), 275-290.

11. T.-Y. Lee, Algebraic relations for multiple zeta values through shuffle product formulas, Ph.D. Dissertation, 2012.

12. N. Nielsen, Die Gammafunktion, Band I; Handbuch der Theorie der Gammafunktion, Band II; Theorie des Integrallogarithmus und verwandter Transzendenten, 1906, Reprinted together as Die Gammafunktion, Chelsea Publishing Co., New York, 1965.

13. Y. Ohno, A generalization of the duality and sum formulas on the multiple zeta values, J. Number Theory, 74(1) (1999), 39-43.

14. J. Pitman, Probability, Springer-Verlag Inc., New York, 1993.

\author{
Yao Lin Ong \\ Department of Accounting and Information System \\ Chang Jung Christian University \\ Gueiren Dist., Tainan City 71101 \\ Taiwan \\ E-mail: ylong@mail.cjcu.edu.tw
}

